\title{
A Robust Visual Tracker Based on DCF Algorithm
}

\author{
Menglei Jin, Weibin Liu* \\ Institute of Information Science \\ Beijing Jiaotong University \\ Beijing 100044, China \\ E-mail: wbliu@bjtu.edu.cn
}

\author{
Weiwei Xing \\ School of Software Engineering \\ Beijing Jiaotong University \\ Beijing 100044, China
}

\begin{abstract}
Since Correlation Filter appeared in the field of video object tracking, it is great popular due to its excellent performance. The Correlation Filter based tracking algorithms are very competitive in terms of accuracy and speed as well as robustness. However, there are still some fields for improvement in the Correlation Filter based tracking algorithms. First, during the training of the classifier, the background information that can be utilized is very limited. Moreover, the introduction of the cosine window further reduces the background information. These reasons reduce the discriminating power of the classifier. This paper introduces more global background information on the basis of the DCF tracker to improve the discriminating ability of the classifier. Then, in some complex scenes, tracking loss is easy to occur. At this point, the tracker will be treated the background information as the object. To solve this problem, this paper proposes a novel re-detection component. Finally, the current Correlation Filter based tracking algorithms use the linear interpolation model update method, which cannot adapt to the object changes in time. This paper proposes an adaptive model update strategy to improve the robustness of the tracker.
\end{abstract}

Keywords-visual tracking; Correlation Filter; background modeling; re-detection and re-search; model update

\section{INTRODUCTION}

Video object tracking is a very important and popular study in the field of computer vision. It has applications in many areas, such as precision guidance in the military field, intelligent transportation and autonomous driving, and human-computer interaction. At present, more and more research results have been achieved in this field. Both tracking accuracy and speed have been greatly improved. However, it still faces many challenges, especially complex scenes such as lighting changes, motion blur, and occlusion [1]-[2].

We focus on single object tracking in this paper. At present, there are two types of methods in the field of object tracking. One is the generative approach, the other is the discriminative approach. It's not unfamiliar to generative models, such as classic mean-shift, particle filtering, kalman filtering and so on. Unlike the generative model, the discriminative model trains a classifier which can effectively distinguish between the object and the background. As far as the current research progress is concerned, the discriminative model is superior to the generation model.

\{Correspending author: Weibin Liu, wbliu@ bjtu.edu.cn\}

DOI reference number: 10.18293/SEKE2019-149
The latest research progress of discriminative approach is Correlation Filter based algorithms. Correlation Filter is simply referred to as CF. Correlation is a concept in the field of signal processing. In real life, many problems can be solved with Correlation functions. It measures the degree of similarity of two signals at a time. Therefore, the more similar the two signals are, the larger the correlation value is. Been inspired by this idea, the MOSSE (Minimum Output Sum of Squared Error) tracker skillfully applies Correlation Filter to video object tracking [3]. Specifically, during the tracking process, we need to train a Correlation Filter template based on the object image block and desired output. When this template is applied to the candidate image blocks, the image block with the largest response value is regarded as the object. Why are Correlation Filter based algorithms capable of achieving so high speeds? This is mainly due to cyclic shift and Fourier transform, which can greatly reduce the computational complexity.

Nevertheless, there are still some problems to be solved in Correlation Filter based algorithms. (1) The problem of insufficient background information. It is well known that negative samples are very important in the training of classifier. However, in order to reduce the boundary effect caused by cyclic shift, the algorithm uses the operation of adding cosine window. This operation not only reduces the proportion of real samples, but also further reduces the background information. (2) The problem of tracking loss and model drift. In complex scenarios, tracking loss often occurs in the process of tracking. Traditional algorithms do not take measures to deal with this situation. (3) The problem of model updating cannot adapt to object changes. Classical algorithms often use fixed update rate to update the model linearly. This updating method has a great defect, it cannot reflect the change of the target in time. It can seriously affect tracking accuracy.

To solve these problems, this paper makes the following contributions. The proposed algorithm is based on DCF tracker. In order to improve the discriminant performance of the classifier, we combine the framework in CACF [4] with the DCF framework. Then, we add the operation of re-detection and research on the basis of the original algorithm flow. Here, redetection depends on tracking confidence index. Finally, we introduce an adaptive model updating strategy, which can adapt to the appearance changes of the object in time. Experiments show that these measures are effective.

\section{Related Work}

Blome et al. first applied Correlation Filter to video object tracking. The MOSSE tracker uses an objective function with a minimum mean square error. Due to the full utilization of the 
Fourier transform, the tracking speed of the algorithm is very fast. Its accuracy rate is $43.1 \%$ while maintaining $615 \mathrm{fps}$. The disadvantage of the MOSSE tracker is that the feature representation is relatively simple, which greatly affects the tracking accuracy. The CSK (Circulant Structure with Kernels) tracker proposed by Henriques et al. solves the tracking problem from the perspective of machine learning [5]. It uses a cyclic shift sampling strategy to solve the problem of insufficient training samples that always exist in object tracking field. However, the shortcoming of the feature representation is still unresolved. Later, the $\mathrm{KCF} / \mathrm{DCF}$ tracker was proposed [6]. It extends the feature representation of the image from the single channel gray features to multichannel HOG (Histogram of Oriented Gradients) feature. Compared with the CSK tracker, the tracking accuracy has increased from $54.4 \%$ to $73.2 \%$. Among them, the difference between KCF (Kernelized Correlation Filter) and DCF (Discriminative Correlation Filter) is use of nuclear techniques. Similarly, the CN (Color Name) tracker extends the feature representation from gray features to color attributes [7]. The $\mathrm{CN}$ feature subdivides the RGB color into 11 common color in life [8]-[9]. To avoid high computational complexity, the algorithm uses the PCA method to process features. Later, Danelljan M et al. proposed the DSST (Discriminative Scale Space Tracker), which solves the multiscale problem in object tracking [10]. During the tracking process, the algorithm designs two independent Correlation Filters to estimate the scale and the position of object. Another tracker that can solve multi-scale problem is SAMF (Scale Adaptive Filter with Feature Integration), which was proposed by Yang L. This algorithm combines the FHOG feature with the $\mathrm{CN}$ feature for the first time. It adopts a multi-scale detection algorithm in the field of object detection. Unlike the DSST tracker, the SAMF tracker [11] can simultaneously estimate the location and scale of the object.

At present, there are already some tracking algorithms that have done work on re-detection. The MOSSE tracker proposes a PSR indicator to measure tracking confidence. Then, LCT (Long-term Correlation Filter Tracker) introduces an additional Correlation Filter in the base of the DSST that can be used to detect object confidence [12]. If the tracking confidence factor does not meet the requirements, the algorithm initiates a research component. The algorithm uses the SVM classification algorithm to relocate. In terms of model updating, the LMCF (Large margin tracking method with circulant feature maps) tracker proposes a robust model updating strategy [13]. The model is updated only when the tracking confidence coefficient reaches the historical mean. Otherwise, the model is not updated. In addition, the ECO tracker uses another more efficient way to perform model updates. The tracking model is updated every few frames [14].

\section{OUR METHOD}

In this section, we detail our proposed object tracking algorithm. Because the algorithm is based on the DCF tracker, we first introduced the basic tracker. Then, we introduced the core of this paper: global background modeling, re-detection component and adaptive model update strategy.

\section{A. The Base Method: DCF tracker}

The introduction of a large number of negative samples can improve the discriminative performance of the classifier during the tracking process. Many tracking algorithms determine the negative samples based on the distance of the object in the two frames. The overlap rate of these negative samples is actually very high. Therefore, the DCF tracker constructs training samples by cyclic shifting of the tracking object. As shown in Equation 1, the DCF tracker uses the regularized least squares classification algorithm to train the classifier.

$$
\min _{w} \sum_{i}\left(f\left(x_{i}\right)-y_{i}\right)^{2}+\lambda\|w\|^{2}
$$

Where $f$ denotes a classification function, $x_{i}$ and $y_{i}$ represent training samples and expected outputs, respectively, $W$ represents the weight of the classifier, and $\lambda$ is a regularization parameter to prevent overfitting. Then we write it in the form of a matrix.

$$
\min _{w} \sum_{i}(X W-y)^{2}+\lambda\|w\|^{2}
$$

In formula 2, $X$ and $y$ represent the matrix form of $x_{i}$ and $y_{i}$, respectively. To minimize the objective function, we take advantage of the nature of the cyclic matrix. The solution can be obtained by setting the derivative to 0 .

$$
\widehat{w}=\frac{\hat{x}^{*} \odot \hat{y}}{\hat{x}^{*} \odot \hat{x}+\lambda}
$$

Then, we introduce a kernel function to solve the problem of insufficient discriminant performance of the linear classifier. The kernel function maps the input data to a nonlinear feature space. Through the mapping, we can get the following expression:

$$
w=\min _{w}\|\phi(X) w-y\|^{2}+\lambda\|w\|^{2}
$$

Here, $w$ satisfies $w \stackrel{w}{=} \sum_{i} \alpha_{i} \phi\left(x_{i}\right)$. Therefore, the above formula can be expressed as:

$$
\alpha=\min _{\alpha}\left\|\phi(X) \phi(X)^{T} \alpha-y\right\|^{2}+\lambda\|w\|^{2}
$$

Through a series of derivations, the solution can be expressed as:

$$
\alpha=F^{-1}\left(\frac{\hat{y}}{\hat{k}^{x x}+\lambda}\right)
$$

Where $k^{x x}$ is the first row of the kernel matrix $k$. The matrix $k$ satisfies $k=\phi(X) \phi(X)^{T}$. In order to locate the tracking object in each subsequent frame, the DCF tracker also uses the cyclic shift to build the samples.

$$
f\left(z_{j}\right)=w^{T} \varphi\left(z_{j}\right)
$$

$z_{j}$ in Equation 7 represents a sample obtained by cyclically shifting the image block $z$, which satisfies $z_{j}=p^{j} z$. We can estimate the position of the object by calculating the detection values of all samples by the following formula.

$$
f(z)=\left(k^{z}\right)^{T} \alpha
$$

Through the diagonalization operation, the above formula can be converted into:

$$
\hat{f}(z)=\hat{k}^{x z} \odot \hat{\alpha}
$$

Where $k^{x z}$ is the nuclear correlation of $x$ and $z$. Repeat this process and the tracking process is complete. 


\section{B. Global background modeling}

In the tracking process of the $\mathrm{KCF} / \mathrm{DCF}$ tracker, the search range is usually 1.5 times the size of the object. However, the operation of the cyclic shift is used in the construction of the sample, which brings the boundary effect. To alleviate the boundary effect, the DCF tracker adds a cosine window. However, the cosine window reduces not only the search range, but also the available background information available during the tracking process. In this paper, we combine the DCF framework with the framework in CACF to increase global context information.

During the training of the Correlation Filter, we sampled around the object. We train the ridge regression classifier using the object and the background image block. To get the closed solution, we regress the expected value of the global background image block to zeros. The objective function is:

$$
\min _{w}\left\|A_{0} w-y\right\|_{2}^{2}+\lambda_{1}\|w\|_{2}^{2}+\lambda_{2} \sum_{i=1}^{k}\left\|A_{i} w\right\|_{2}^{2}
$$

Where $A_{0}$ represents the cyclic matrix formed by the object image block, $A_{i}$ represents the cyclic matrix constructed by the global background image block, and $\lambda_{1}$ and $\lambda_{2}$ are regularization parameters. In the objective function, the expected output value of the object image block is y. We recombine the object image block and the background image block to form a new matrix. This formula can be rewritten as:

$$
f_{p}(w, B)=\|B w-\bar{y}\|_{2}^{2}+\lambda_{1}\|w\|_{2}^{2}
$$

Here, $B$ denotes a new matrix formed by combining the global background image block and the object, and $\bar{y}$ denotes a label vector obtained by expanding the label $y$ with 0 . $B$ and $\bar{y}$, satisfy,

$$
B=\left[\begin{array}{c}
A_{0} \\
\sqrt{\lambda_{2}} A_{1} \\
\vdots \\
\sqrt{\lambda_{2}} A_{k}
\end{array}\right] \text { and } \bar{y}=\left[\begin{array}{c}
y \\
0 \\
\vdots \\
0
\end{array}\right]
$$

Since $f_{p}(w, B)$ is a convex function, by deriving the objective function and setting the derivative to 0 , a closed solution for $\mathrm{w}$ can be obtained:

$$
w=\left(B^{T} B+\lambda_{1} I\right)^{-1} B^{T} \bar{y}
$$

Using the property of the cyclic matrix, the above formula can eventually be converted to:

$$
\begin{gathered}
w=\left[F \operatorname { d i a g } \left(\hat{\alpha}_{0}^{*} \odot \hat{\alpha}_{0}+\lambda_{1}+\lambda_{2} \sum_{i=1}^{k} \hat{\alpha}_{i}^{*} \odot\right.\right. \\
\left.\left.\hat{\alpha}_{i}\right) F^{H}\right]^{-1} F \operatorname{diag}\left(\hat{\alpha}_{0}^{*} \odot \hat{y}\right)
\end{gathered}
$$

By performing a Fourier transform on $w$, we can get:

$$
\widehat{w}=\frac{\hat{\alpha}_{0}^{*} \odot \hat{y}}{\hat{\alpha}_{0}^{*} \odot \hat{\alpha}_{0}+\lambda_{1}+\lambda_{2} \sum_{i=1}^{k} \hat{\alpha}_{i}^{*} \odot \hat{\alpha}_{i}}
$$

The next step is object detection. The response map can be obtained by convoluting the learned filter with the image block $z$ (search window) in the next frame. Much like the DCF tracker, the detection formula for solving the response map can be expressed as:

$$
\hat{r}_{d}=\hat{z} \odot \hat{\alpha}_{0}^{*} \odot \hat{\alpha}_{0}+\sqrt{\lambda_{2}} \sum_{i=1}^{k} \hat{z} \odot \hat{\alpha}_{i}^{*} \odot \hat{\alpha}_{i}
$$

\section{Re-detection and re-search components}

In the traditional Correlation Filter tracking algorithms, the principle of tracking is to apply the Correlation Filter template to the detect image blocks, and regard the position of the peak in the response map as the position of the object. However, there is a drawback in such a detection method which the position with the largest response value does not always track the actual position of the object, especially in some complicated scenarios. Therefore, it is likely to bring about model pollution with using this method for position estimation directly, which affects the accuracy of the tracking algorithm. In this paper, we introduce a re-detection and re-search operation to improve this problem. We used the APCE indicator to determine the tracking performance of the current frame [13]. The APCE calculation method is as follows:

$$
A P C E=\frac{|\max (\phi(t))-\min (\phi(t))|^{2}}{\operatorname{mean}\left(\sum_{m, n}\left(g_{m, n}-\min (\phi(t))^{2}\right)\right.}
$$

Where $\phi(t)$ represents the response map of the t-th frame. When the APCE indicator does not reach the historical average, the re-detection component is turned on. We re-search for the object at the position corresponding to the other peaks of the response map. Then, the response maps obtained by the correlation filter template at all candidate image blocks are respectively calculated. Finally, we determine the image block with the largest response value as the object.

\section{Adaptive model update strategy}

During the tracking process, the object often undergoes various changes. Therefore, the tracking algorithm needs to update the model in time to adapt to this change to improve the robustness of the tracking algorithm. This is very challenging. In the classic Correlation Filter based tracking algorithms, both $\mathrm{KCF}$ and DSST tracker use the following linear interpolation update method.

$$
\begin{aligned}
& \hat{x}^{n}=(1-\gamma) \hat{x}^{n-1}+\gamma \hat{x} \\
& \hat{\alpha}^{n}=(1-\gamma) \hat{\alpha}^{n-1}+\gamma \hat{\alpha}
\end{aligned}
$$

Where $n$ is the sequence number of the current frame, $\hat{x}$ is the target representation model represented by the predicted position image block, and $\hat{\alpha}$ is the classifier parameter, $\gamma$ represents the model update rate. Currently, the model update rate is determined by empirical values and is constant. The larger the value of $\gamma$, the faster the model update rate. Conversely, the smaller the value of $\gamma$, the slower the model update rate. When a large change occurs in the object, such as posture change and in-plane rotation, etc., $\gamma$ should be selected as a larger value. When the tracking environment changes greatly and the tracking object does not change much, $\gamma$ should be selected as a smaller value. However, when $\gamma$ is too large, the update rate is too fast and it is easy to cause model drift. In summary, setting the model update rate to a fixed value is not appropriate because it does not accurately reflect changes in the object. Moreover, this update method easily leads to over-fitting of the model to several frames 
of images. For example, the updated model is particularly sensitive to occlusion and deformation. In this paper, we propose an adaptive model update strategy. Here, we introduce a penalty coefficient $\xi$ to control the model update rate. $\xi$ is determined by the following formula.

$$
\xi=\frac{\max (\phi(t))}{\max (\{\phi(1), \phi(2), \phi(3), \cdots \phi(t-1)\})}
$$

\section{EXPERIMENT RESULTS}

\section{A. Dataset and evaluation index}

In order to test the performance of the algorithm, this paper uses the OTB-2013 and OTB-2015 datasets, which are very popular in the field of video object tracking. Prior to the advent of OTB-2013, there was no recognized database here, so the tracking performance of the algorithm could not be accurately tested and compared. The significance of OTB-2013 is profound, which greatly promotes the development of tracking algorithms. OTB-2013 includes a total of 50 video sequences, while OTB2015 extends it from 50 video sequences to 100 video sequences. The datasets contain 11 tracking difficulties: illumination changes, scale changes, occlusion, deformation, motion blur, fast motion, in-plane rotation, out-of-plane rotation, out-of-field, background clutter, low resolution. There are 26 gray sequences and 74 color sequences in 100 video sequences. The tracking targets that appear in OTB-2015 dataset include 36 entities, 26 faces/headers, and a total of 58897 frames. The length of the video sequence includes short-term and long-term, and the longest video sequence has more than 3,000 frames.

The OTB-2013 and OTB-2015 datasets provide two evaluation indicators: precision and success rate. Precision is an indicator used to measure the center pixel position deviation. As shown in Figure 1, the estimated tracking frame of the algorithm is $S^{\prime}$, and its central pixel position is $\left(x^{\prime}, y^{\prime}\right)$. The actual tracking frame is $S$, and its center pixel position is $(x, y)$. Use the Euclidean distance when calculating the center pixel deviation. Typically, the center pixel deviation threshold is set to 20 pixels. The success rate is calculated as follows:

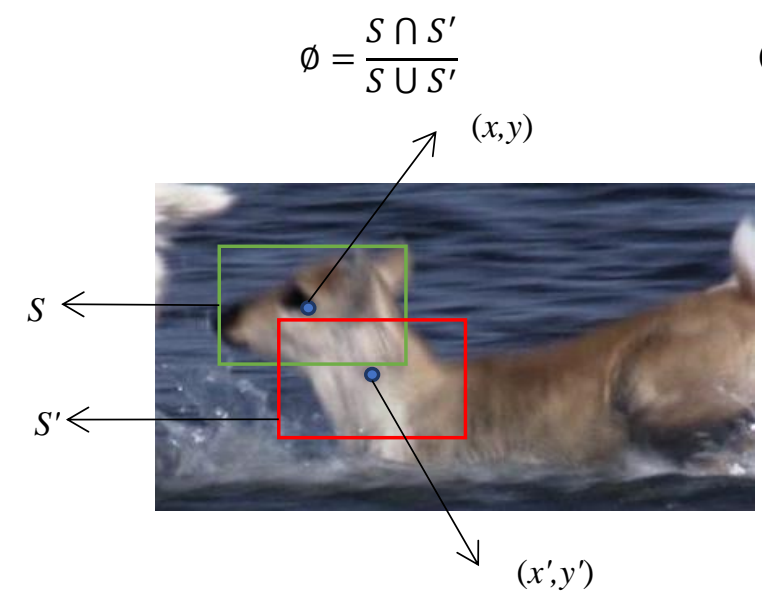

Figure 2. Ground truth and actual tracking results comparison chart

\section{B. Parameter setting and algorithm flow chart}

In practice, we implemented the proposed algorithm using the MATLAB language. In this paper, the kernel function type is set to a linear kernel, the image block padding is set to $2, \lambda_{1}$ is set to $1 e-4, \lambda_{2}$ is set to 26 , and the linear interpolation factor for adaptation is set to 0.012 . When calculating the HOG feature, the cellSize is set to 4 and the orientation is set to 9 .

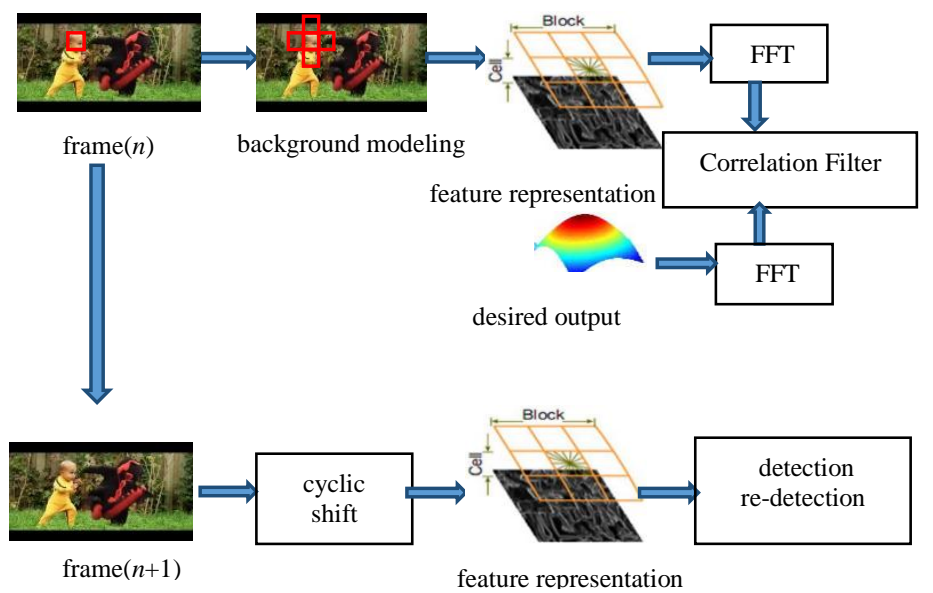

Figure 2. Algorithm flow chart

Figure 2 shows in detail the flow chart of the tracking algorithm proposed in this paper. Compared with the traditional Correlation Filter based algorithms, our proposed DCF_BM tracker has improved the training process of the Correlation Filter. On the negative samples of the training, not only the image blocks after the object cyclic shift are used, but also the image blocks around the object are also taken into consideration. In addition, an adaptive model update strategy is proposed in this paper to better adapt to object changes. Additionally, the redetection and relocation components are also used in the DCF_BM tracker.

\section{Performance testing and analysis}

In this section, we compare the proposed algorithm with some of the Correlation Filter based trackers that have appeared in recent years. Because the KCF tracker has been significantly better than traditional trackers including Struck [15], TLD [16] and MEEM [17], we only compared algorithms include CSK, $\mathrm{KCF}, \mathrm{SAMF}$, DSST and Staple tracker [18].

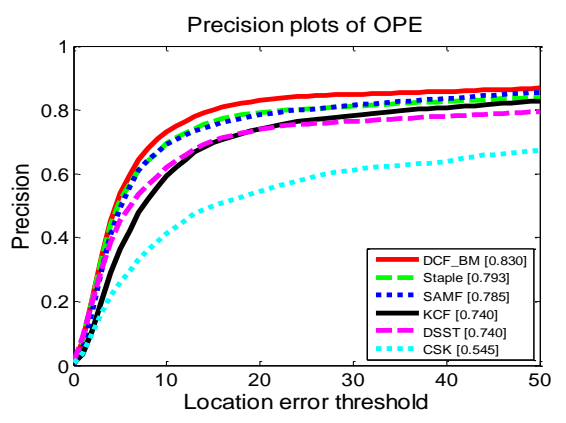

(a) 


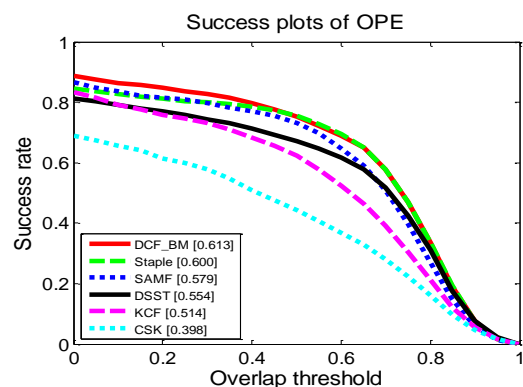

(b)

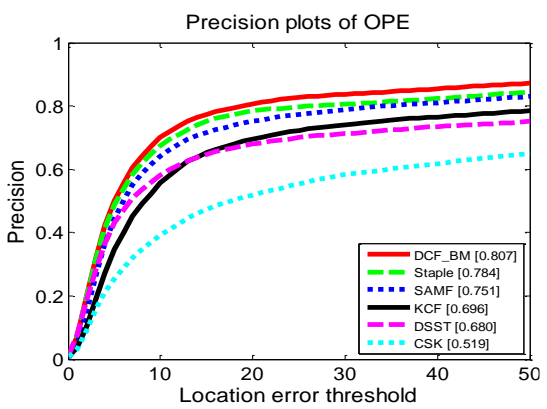

(c)

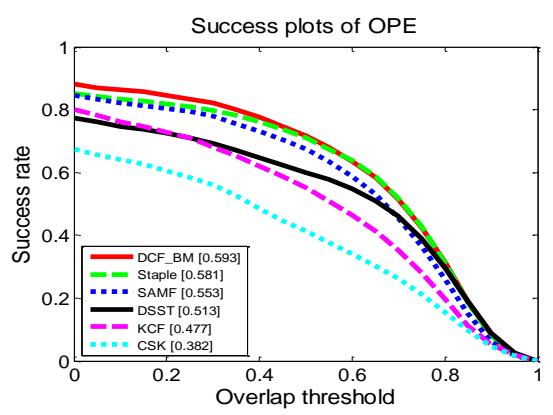

(d)

Figure 3. Experimental results on OTB-2013 and OTB-2015

The experimental results of these algorithms on the OTB2013 dataset are shown in (a) and (b) of the Figure 3. It can be seen that the DCF_BM tracker proposed in this paper has obtained the optimal tracking result on the OTB_2013 dataset. The precision is $83.0 \%$ and the success rate is $61.3 \%$. Compared to the baseline tracker, it achieves significant improvements in accuracy and success rate (both 9\%). The experimental results on the OTB-2015 dataset are shown in Figures (c) and (d) of the Fig. 3. We can find that the DCF_BM tracker proposed in this chapter still achieves the best performance. It has an accuracy of $80.7 \%$ and a success rate of $59.3 \%$. Compared with the classic $\mathrm{KCF}$ tracker, it has increased by $11.0 \%$ and $16.0 \%$ respectively in terms of accuracy and success rate. From these comparisons, one can conclude that the work done in this paper is effective and can improve tracking performance.

In addition, by comparing the experimental results, the Staple tracker ranks second in each result graph. This is mainly because the tracker combines HOG features and statistical color histograms. The response map is linearly weighted to obtain the final response map. The boundary effect is inherently a defect in the Correlation Filter based tracking framework, while the pixellevel color probability features are not affected by this factor. So, from this perspective, the DAT [19] in the Staple tracker mitigates the boundary effect. However, the Staple tracker roughly uses the linear weighting method in the specific implementation. The Correlation Filter response occupies a weight of 0.7 , which plays a major role, while the color probability response occupies a weight of 0.3 , which plays a supporting role. However, this simple weighting method has an obvious disadvantage in that it cannot adaptively determine the weights of the correlation filter response and the color probability response.

In order to further verify the performance of the DCF_BM tracker proposed in this paper under different challenges, the attribute analysis on the OTB-2015 dataset was also carried out. The tracking performance of our proposed tracker and several other advanced trackers is shown in Tab. 1 and Tab. 2. It can be seen that the DCF_BM tracker proposed in this paper achieves the best performance in almost all attributes, in terms of precision or success rate, except LR (low resolution). In addition, the performance of the DCF_BM tracker is improved under various attributes compared to the standard $\mathrm{KCF}$ tracker, which is due to the use of background modeling.

Especially in occlusion (precision $73.8 \%$ vs. $65.1 \%$ ) and fast motion (precision $74.7 \%$ vs. $62.1 \%$ ) scenes, there are significant improvements in tracker's performance. It is mainly due to the application of re-detection and re-search components. When there is an abnormality in the response map, such as in fast motion and occlusion scenarios, we can try to expand the search area and re-detect the tracking object.

\section{CONCLUSION}

In this paper, a tracking framework based on DCF tracker is proposed. The introduction of global background information solves the problem that the background information in the traditional Correlation Filter framework is very limited, thereby improving the discriminating ability of the classifier. The DCF tracker adopts the model updating method of linear interpolation, which is difficult to adapt to the change of the object. The DCF_BM tracker proposed in this paper introduces a penalty coefficient that controls the update rate of the model, which significantly improves this shortcoming.

TABLE I. AVERAGE PRECISION SCORE ON OTB-2015 DATASET

\begin{tabular}{cccccc}
\hline attribute & DCF BM & Staple & DSST & KCF & SAMF \\
\hline IV(38) & 0.817 & 0.791 & 0.721 & 0.719 & 0.715 \\
OPR(63) & 0.762 & 0.738 & 0.644 & 0.677 & 0.739 \\
SV(64) & 0.757 & 0.727 & 0.638 & 0.633 & 0.705 \\
OCC(49) & 0.738 & 0.726 & 0.597 & 0.630 & 0.726 \\
DEF(44) & 0.779 & 0.748 & 0.542 & 0.617 & 0.686 \\
MB(29) & 0.738 & 0.707 & 0.567 & 0.601 & 0.655 \\
FM(39) & 0.747 & 0.697 & 0.552 & 0.621 & 0.654 \\
IPR(51) & 0.799 & 0.770 & 0.691 & 0.701 & 0.721 \\
OV(14) & 0.708 & 0.661 & 0.481 & 0.501 & 0.628 \\
BC(31) & 0.785 & 0.766 & 0.704 & 0.713 & 0.689 \\
LR(9) & 0.638 & 0.631 & 0.567 & 0.560 & 0.685 \\
\hline
\end{tabular}


TABLE II.

AVERAGE SuCCESS SCORE ON OTB-2015 DATASET

\begin{tabular}{cccccc}
\hline attribute & DCF_BM & Staple & DSST & KCF & SAMF \\
\hline IV(38) & 0.615 & 0.598 & 0.558 & 0.479 & 0.534 \\
OPR(63) & 0.549 & 0.534 & 0.470 & 0.453 & 0.536 \\
SV(64) & 0.537 & 0.525 & 0.468 & 0.394 & 0.495 \\
OCC(49) & 0.560 & 0.548 & 0.453 & 0.443 & 0.540 \\
DEF(44) & 0.566 & 0.554 & 0.420 & 0.436 & 0.509 \\
MB(29) & 0.567 & 0.546 & 0.469 & 0.459 & 0.525 \\
FM(39) & 0.571 & 0.537 & 0.447 & 0.459 & 0.507 \\
IPR(51) & 0.564 & 0.552 & 0.502 & 0.469 & 0.519 \\
OV(14) & 0.515 & 0.481 & 0.386 & 0.393 & 0.480 \\
BC(31) & 0.585 & 0.574 & 0.523 & 0.498 & 0.525 \\
LR(9) & 0.417 & 0.418 & 0.383 & 0.307 & 0.430 \\
\hline
\end{tabular}

\section{ACKNOWLEDGMENT}

This research is partially supported by National Natural Science Foundation of China (No.61876018).

\section{REFERENCES}

[1] Wu Y, Lim J, Yang M H. Object Tracking Benchmark[J]. IEEE Transactions on Pattern Analysis \& Machine Intelligence, 2015, 37(9):1834-1848.

[2] Wu Y, Lim J, Yang M H. Online Object Tracking: A Benchmark[C]// Computer Vision and Pattern Recognition. IEEE, 2013:2411-2418.

[3] Bolme D S , Beveridge J R , Draper B A, et al. Visual object tracking using adaptive correlation filters[J]. 2010.

[4] Mueller M, Smith N, Ghanem B, et al. Context-Aware Correlation Filter Tracking[C]// Computer Vision and Pattern Recognition. IEEE, 2017: 1387-1395.

[5] João F. Henriques, Caseiro R , Martins P, et al. Exploiting the Circulant Structure of Tracking-by-Detection with Kernels[M]// Computer Vision - ECCV 2012. Springer Berlin Heidelberg, 2012.

[6] Henriques J F , Caseiro R , Martins P, et al. High-Speed Tracking with Kernelized Correlation Filters[J]. IEEE Transactions on Pattern Analysis and Machine Intelligence, 2015, 37(3):583-596.

[7] Danelljan M , Khan F S , Felsberg M, et al. Adaptive Color Attributes for Real-Time Visual Tracking[C]// 2014 IEEE Conference on Computer Vision and Pattern Recognition. IEEE, 2014.

[8] Weijer J V D , Schmid C, Verbeek J . Learning Color Names from RealWorld Images[C]// 2007 IEEE Conference on Computer Vision and Pattern Recognition. IEEE Computer Society, 2007.

[9] Van d W J , Schmid C, Verbeek J , et al. Learning Color Names for RealWorld Applications[J]. IEEE Transactions on Image Processing, 2009, 18(7):1512-1523.

[10] Danelljan M, Häger G, Khan F, et al. Accurate scale estimation for robust visual tracking $[\mathrm{C}] / /$ British Machine Vision Conference, Nottingham, September 1-5, 2014. BMVA Press, 2014.

[11] Li Y, Zhu J . A Scale Adaptive Kernel Correlation Filter Tracker with Feature Integration[J]. 2014.

[12] Ma C, Yang X, Zhang C, et al. Long-term correlation tracking[C]// Computer Vision and Pattern Recognition., 2015: 5388-5396.

[13] Wang M, Liu Y, Huang Z. Large Margin Object Tracking with Circulant Feature Maps[J]. 2017:4800-4808.

[14] Danelljan M , Bhat G, Khan F S , et al. ECO: Efficient Convolution Operators for Tracking[J]. 2016.

[15] Hare S , Saffari A, Torr P H S . Struck: Structured output tracking with kernels[J]. 2011.

[16] Kalal Z, Mikolajczyk K, Matas J. Tracking-Learning-Detection.[J]. IEEE Trans Pattern Anal Mach Intell, 2012, 34(7):1409-1422.

[17] Zhang J, Ma S, Sclaroff S. MEEM: Robust Tracking via Multiple Experts Using Entropy Minimization[M]// Computer Vision - ECCV 2014. Springer International Publishing, 2014:188-203.

[18] Bertinetto L, Valmadre J, Golodetz S, et al. Staple: Complementary learners for real-time tracking[C]//Proceedings of the IEEE Conference on Computer Vision and Pattern Recognition. 2016: 1401-1409.
[19] Possegger H, Mauthner T , Bischof H . In defense of color-based modelfree tracking $[\mathrm{C}] / /$ Computer Vision and Pattern Recognition. IEEE Computer Society, 2015. 